\title{
A Novel Dual-Polarization Total Power Radiometer with Single Channel
}

\author{
Yiming Niu, Can Cui, Guo Yang, and Wen Wu \\ Ministerial Key Laboratory of JGMT, Nanjing University of Science and Technology, Nanjing 210014, China \\ Correspondence should be addressed to Guo Yang; yangguol10@vip.sina.com
}

Received 29 August 2013; Accepted 13 November 2013; Published 13 February 2014

Academic Editors: C. W. Chiou and H. L. Hartnagel

Copyright (C) 2014 Yiming Niu et al. This is an open access article distributed under the Creative Commons Attribution License, which permits unrestricted use, distribution, and reproduction in any medium, provided the original work is properly cited.

\begin{abstract}
A dual-polarization total power millimeter wave (MMW) radiometer with single channel was proposed in this paper. It completed the subtraction of two orthogonally polarized signals in the RF front end. The system used one channel to achieve the function of two channels. On the basis of discussing the theory and the configuration of the system, the performance of the system and the ability to identify the false target were analyzed and simulated. The results showed the radiometer could solve the problem that the performance of dual-polarization radiometer becomes weak because of gain inconformity with two channels. The system was cheap and small and could identify the false target which has different apparent temperatures at vertical- and horizontal-polarized radiations.
\end{abstract}

\section{Introduction}

Passive millimeter wave (MMW) detecting technique is to scan and detect the target area by using MMW radiometer. The target will be detected, identified, and located based on the different intensity levels of the target and background. Since MMW radiometer can easily identify metal and nonmetal, it can be applied in searching and rescuing cars, aircraft navigation, and safety checking in severe environment. One of its applications is in the terminal guidance of smart ammunition [1-3].

False targets like pond which has similar MMW radiation characteristic with metal targets are usually met in passive MMW detecting experimental study. Their MMW radiation temperature is lower than background temperature, so they can hardly be distinguished by general singlepolarization millimeter wave radiometer with single channel. A Ka band dual-polarization direct detection total power MMW radiometer is designed in the literature [4]; this system receives targets and background radiation signal's horizontal polarization component and vertical polarization component through dual-channel receiver; after comparing the differences, the system detects and identifies some false targets whose level and polarization have great difference in temperature. This project can solve false target problem like ponds easily, but it has a high demand on the consisting of the two receivers which can hardly be guaranteed at current technologic level; besides, using two receivers leads to the huge volume and high cost.

In this paper, sum-difference network technology in monopulse antenna [5] is applied to MMW dual-polarization radiometer; then a new dual-polarization total power MMW radiometer system with single channel is designed; it not only finishes the subtraction of two orthogonal polarization signals through radio-frequency head but also makes targets' detection and identification be achieved by using single channel. This study can solve the performance deterioration problem of dual-polarization MMW radiometer which led by the channel gain's inconsistency of dual-channel receiver well, it is small in size and cost low.

\section{System Configuration and Working Principle}

In one-dimensional scanning passive millimeter wave detection application, the system detects, recognizes, and locates target according to the detected millimeter wave noise temperature variation. The output signal variation of radiometer is the useful signal needed by the system; therefore, there is a 


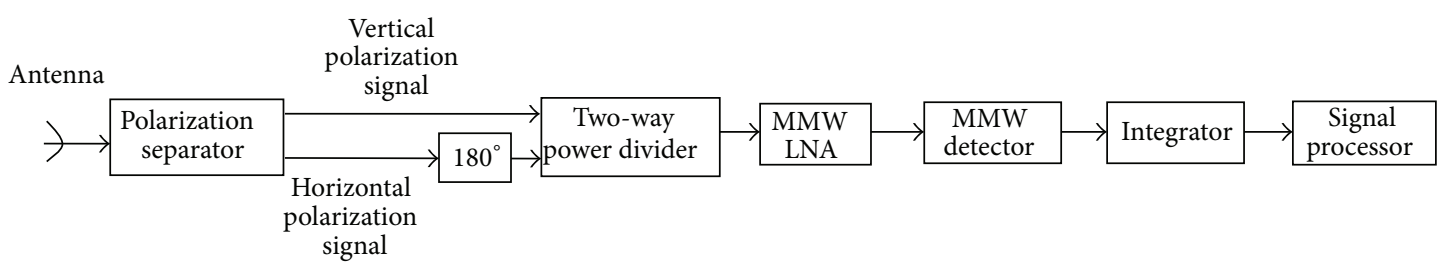

FIgURE 1: Configuration of the dual-polarization total power millimeter wave radiometer with single channel.

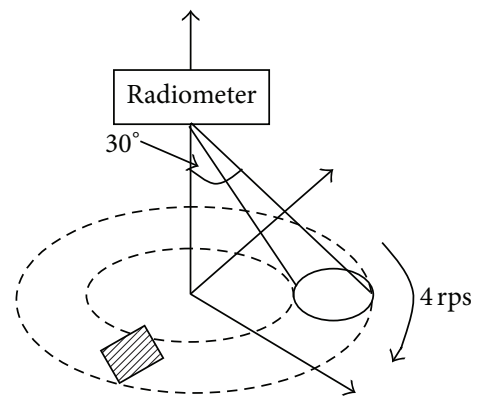

FIGURE 2: Diagram of the principle of the system.

circuit; removes DC component in video amplifier circuit that the AC signal left is useful. It detects and recognizes target according to the output signal amplitude.

2.1. System Configuration. The designed dual-polarization total power MMW radiometer with single channel in this paper is shown in Figure 1. The MMW signal radioed by the target will enter into polarization separator to be separated into horizontal polarization signal and vertical polarization signal after being received by antenna; then the horizontal polarization signal will phase shift $180^{\circ}$ and enter into power divider; together with vertical polarization signal, the two signals will composite one signal. Through amplifying by MMW LNA, detecting by MMW detector, and integrating, the signal will be processed by signal processor; finally, it finishes positing the detection and identification of the target.

2.2. Working Principle of the System. The MMW radiometer system designed in this paper does the conical scan on ground with $30^{\circ}$ angle of declination which is perpendicular to ground; scanning cycle is $4 \mathrm{rps}$, as shown in Figure 2.

When system works, the horizontal polarization signal will phase shift $180^{\circ}$ through power divider and the two signals finish the subtraction between vertical polarization channel signal and horizontal polarization channel signal in radio-frequency head. In order to achieve the function of this system, power divider is designed into unequal two-way power dividers. Power output formula is $\mathrm{P} 1=1.6 \mathrm{P} 2$; $\mathrm{P} 1$ is horizontal polarization signal and $\mathrm{P} 2$ is vertical polarization signal.

\section{Analysis of System Performance}

3.1. Output Signal to Noise Ratio (SNR). When antenna beam is not on the target, we can consider that the antenna
TABLE 1: Radiation temperature of object.

\begin{tabular}{lc}
\hline Parameter & Apparent temperature \\
\hline $\begin{array}{l}\text { Apparent temperature in } \\
\text { horizontal polarization of metal } \\
\text { Apparent temperature in vertical } \\
\text { polarization of metal }\end{array}$ & $50 \mathrm{~K}$ \\
$\begin{array}{l}\text { Apparent temperature in } \\
\text { horizontal polarization of pond } \\
\text { Apparent temperature in vertical } \\
\text { polarization of pond }\end{array}$ & $50 \mathrm{~K}$ \\
$\begin{array}{l}\text { Apparent temperature in } \\
\text { horizontal polarization of } \\
\text { background }\end{array}$ & $140 \mathrm{~K}$ \\
$\begin{array}{l}\text { Apparent temperature in vertical } \\
\text { polarization of background }\end{array}$ & $170 \mathrm{~K}$ \\
\hline
\end{tabular}

TABLE 2: Radiation temperature difference of target.

\begin{tabular}{lcc}
\hline Parameter & $\begin{array}{c}\text { Target full of } \\
\text { antenna beam }\end{array}$ & $\begin{array}{c}\text { Target full of } \\
60 \% \\
\text { antenna beam }\end{array}$ \\
\hline $\begin{array}{l}\text { Temperature difference between } \\
\text { metal and background }\end{array}$ & $36 \mathrm{~K}$ & $21.6 \mathrm{~K}$ \\
$\begin{array}{l}\text { Temperature difference between } \\
\text { pond and background }\end{array}$ & $10.5 \mathrm{~K}$ & $6.3 \mathrm{~K}$ \\
\hline
\end{tabular}

TABLE 3: Output SNR.

\begin{tabular}{lcc}
\hline Parameter & $\begin{array}{c}\text { Target full of } \\
\text { antenna beam }\end{array}$ & $\begin{array}{c}\text { Target full of } \\
60 \% \text { antenna } \\
\text { beam }\end{array}$ \\
\hline Scanning metal target & 36 & 21.6 \\
Scanning pond & 10.5 & 6.3 \\
\hline
\end{tabular}




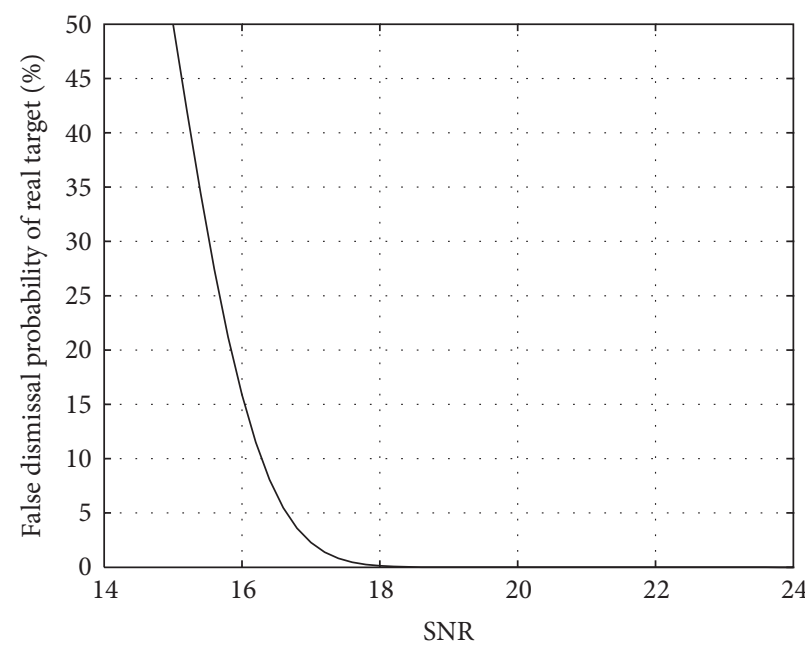

(a) False dismissal probability of real target

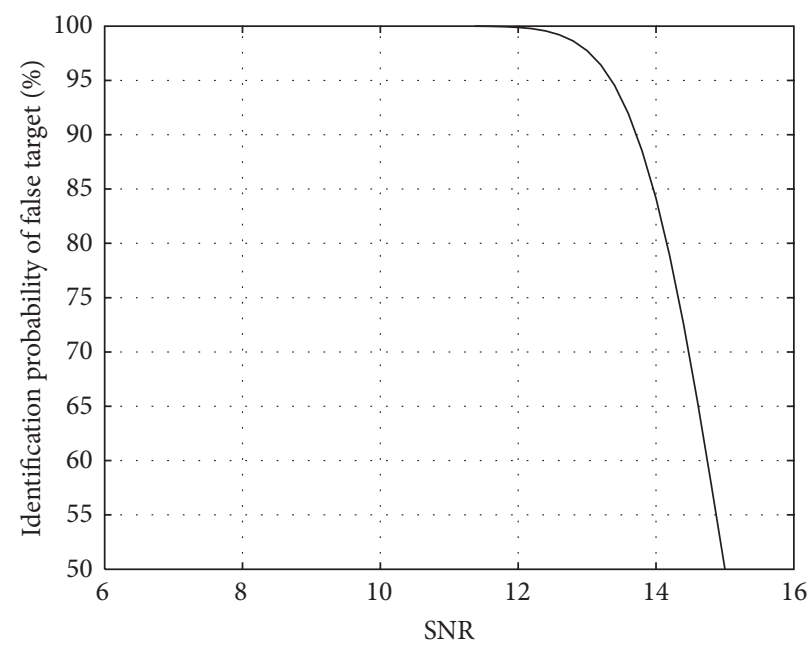

(b) Identification probability of false target

FIGURE 3: Diagram of the system detection probability in limiting cases.

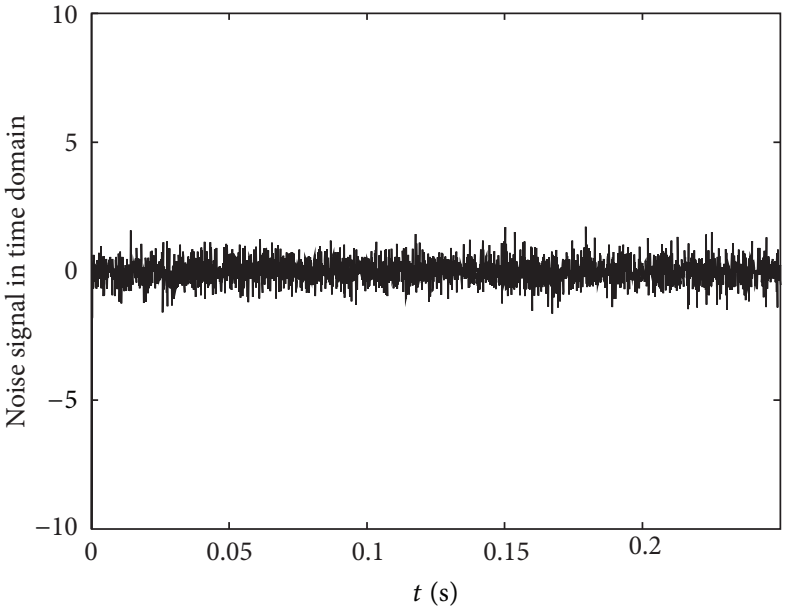

(a)

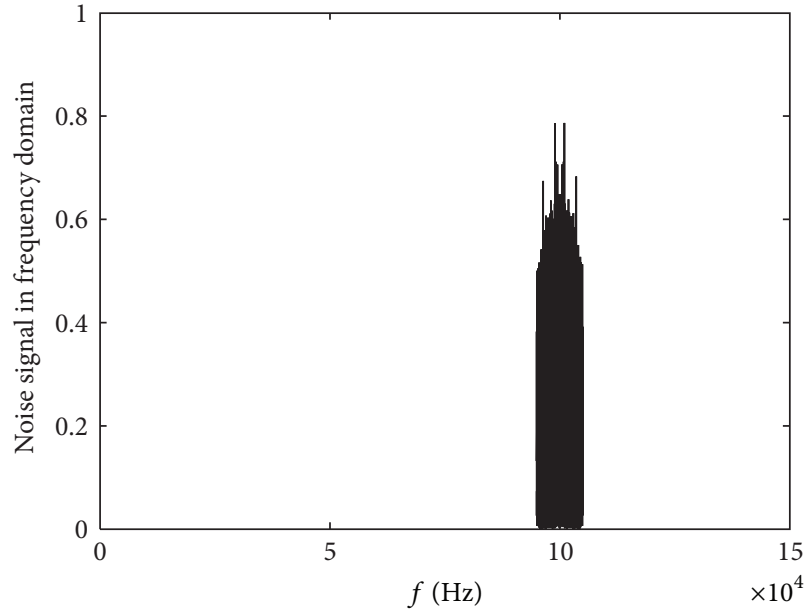

(b)

Figure 4: Produced band-limited Gaussian white noise.

receives is background temperature of MMW noisy. Effect by atmospheric attenuation can be neglected. So, background antenna temperature is $T_{A g}=T_{g A P}\left(T_{g A P}\right.$ is background apparent temperature).

When antenna beam is on the target, the antenna beam solid angle which was taken up by the target could be either greater or less than the solid angle of main beam of antenna. While general detection is applied, antenna gain is higher, antenna beam is narrow and the antenna beam solid angle taken by the target is not big. So we can consider that the antenna receives is just background MMW radiation outside the main beam; antenna temperature of the target is [6]

$$
T_{A T}= \begin{cases}\eta_{m} T_{A P}+\left(1-\eta_{m}\right) T_{A g}, & \Omega_{T} \geq \Omega_{M}, \\ \eta_{m} \frac{\Omega_{T}}{\Omega_{M}} T_{A P}+\left(1-\eta_{m} \frac{\Omega_{T}}{\Omega_{M}}\right) T_{A g}, & \Omega_{T}<\Omega_{M},\end{cases}
$$

where $\eta_{m}$ is efficiency of Antenna main beam, $\Omega_{M}$ is solid angle of antenna main beam, $\Omega_{T}$ is solid angle of antenna beam which was taken by target, and $T_{A P}$ is target apparent temperature.

Signals combine into one way after pass phase shift and power divider; then the output temperature is

$\Delta T_{A}$

$$
= \begin{cases}\frac{\eta_{l} \eta_{m}}{L}\left[\left(T_{A P 1}-T_{A g}\right)-\frac{\left(T_{A P 2}-T_{A g}\right)}{1.6}\right], & \Omega_{T} \geq \Omega_{M}, \\ \frac{\Omega_{T} \eta_{l} \eta_{m}}{\Omega_{M} L}\left[\left(T_{A P 1}-T_{A g}\right)-\frac{\left(T_{A P 2}-T_{A g}\right)}{1.6}\right], & \Omega_{T}<\Omega_{M},\end{cases}
$$

where $T_{A P 1}$ is horizontal polarization apparent temperature of target, $T_{A P 2}$ is vertical polarization apparent temperature 


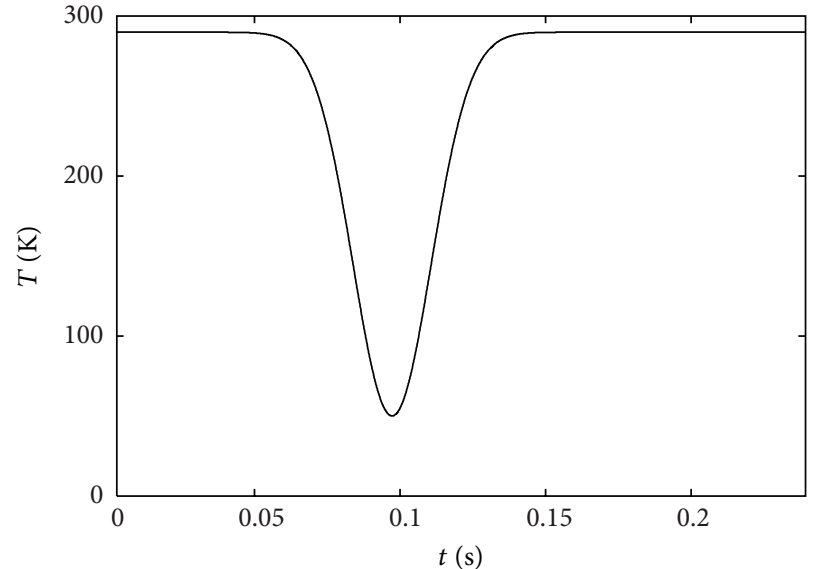

(a) Simulation of horizontal polarization input temperature of metal target

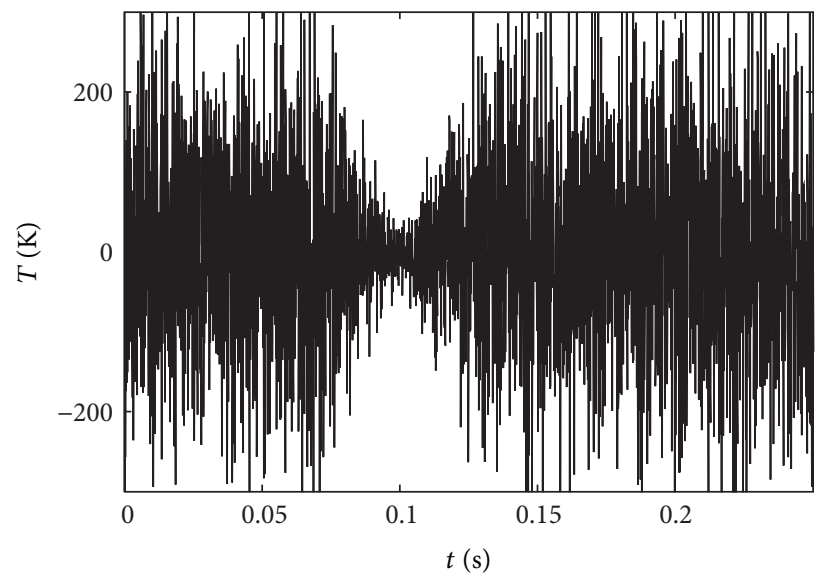

(c) Input signal of metal target in horizontal polarization

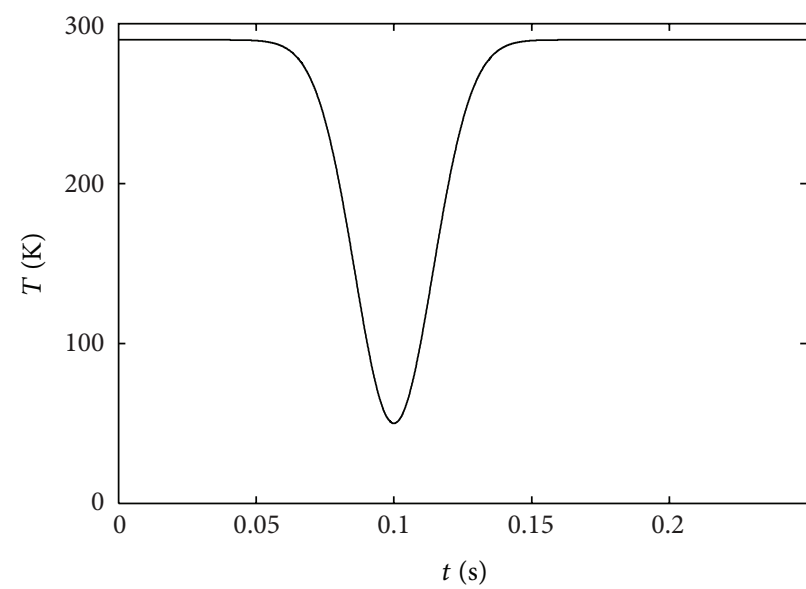

(b) Simulation of vertical polarization input temperature of metal target

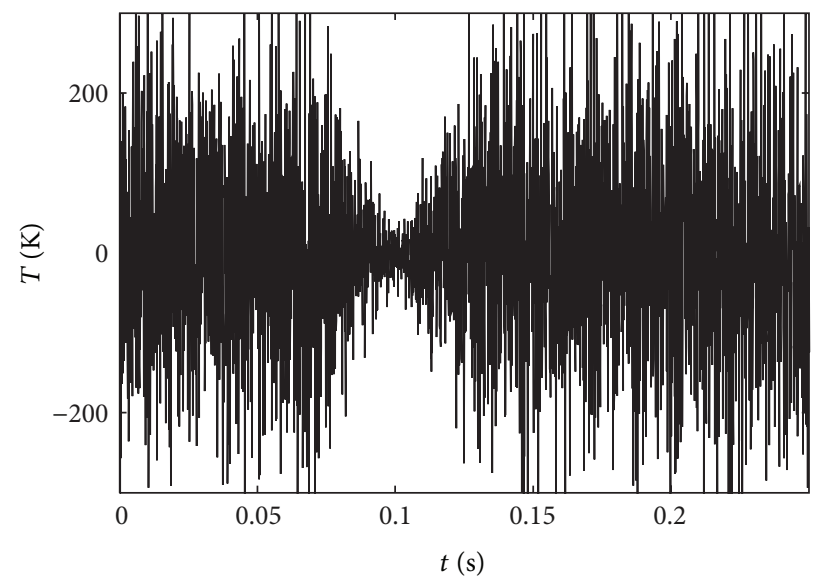

(d) Input signal of metal target in vertical polarization

FIgURE 5: RF input signal of metal target.

of target, $\eta_{l}$ is radiant efficiency of antenna, and $L$ is loss of input circuit.

In the situation that weather is clear and dip, angle of radiometer is $30^{\circ}$ and the apparent temperature of background, metal target, and pond is shown in Table 1 [7].

The system efficiency $\eta=\eta_{l} \eta_{m} / L=0.4$; when the antenna beam width is $3^{\circ}$, the target is full of $60 \%$ of the antenna beam; radiation temperature difference of target calculated by formula (2) is shown in Table 2.

Output SNR can be worked out by SNR formula which is SNR $=\Delta V / \delta=\Delta T_{A} / \Delta T_{\min }$, with radiometer sensitivity $\Delta T_{\min }=1 \mathrm{~K}$ already known, as shown in Table 3 .

According to analysis on Table 3 , due to power divider, great difference in temperature on metal target is produced and shown as a higher crest signal; then metal target and false target pond can be distinguished directly through the judgment on output SNR.

The probability density function which system output obeys is given in [8] setting threshold as 15 , we can get the diagram which shows false dismissal probability of real target in limiting cases and identification probability of false target varies with system output SNR. As shown in Figure 3, system can distinguish between real target and false target effectively.

3.2. Maximum Detection Range of the System. The system maximum detection range is formula (3); $\mathrm{SNR}_{\min }$ is the minimum input SNR needed in the system which needs to reach false-alarm time requirements [6]:

$$
R_{\max }=\left(\frac{A_{T} \Delta T_{A}}{\Omega_{M} \Delta T_{\min } \mathrm{SNR}_{\min }}\right)^{1 / 2}
$$

where $\mathrm{SNR}_{\min }$ is the minimum signal to noise ratio, $\Delta T_{\text {min }}$ is the sensitivity of system, and $A_{T}$ is the equivalent area of target.

When system false-alarm time demand is not less than $60 \mathrm{~s}$, and according to relational graph between false-alarm time and SNR [9], we can get that $\mathrm{SNR}_{\min } \geq 7$; let $\mathrm{SNR}_{\min }=7$ and antenna beam width is $3^{\circ}$; then $R_{\max }=160 \mathrm{~m}$; finally we can get that system maximum detection range is $160 \mathrm{~m}$. 


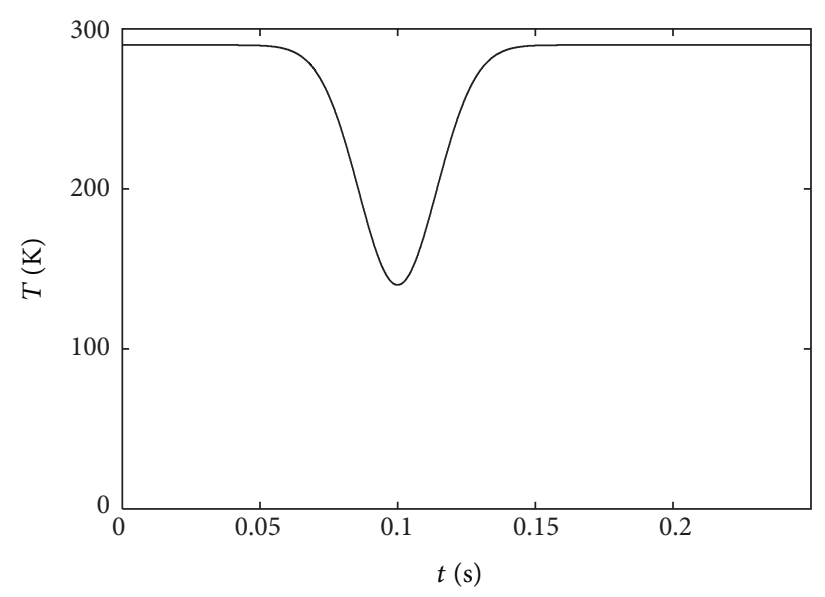

(a) Simulation of horizontal polarization input temperature of pond

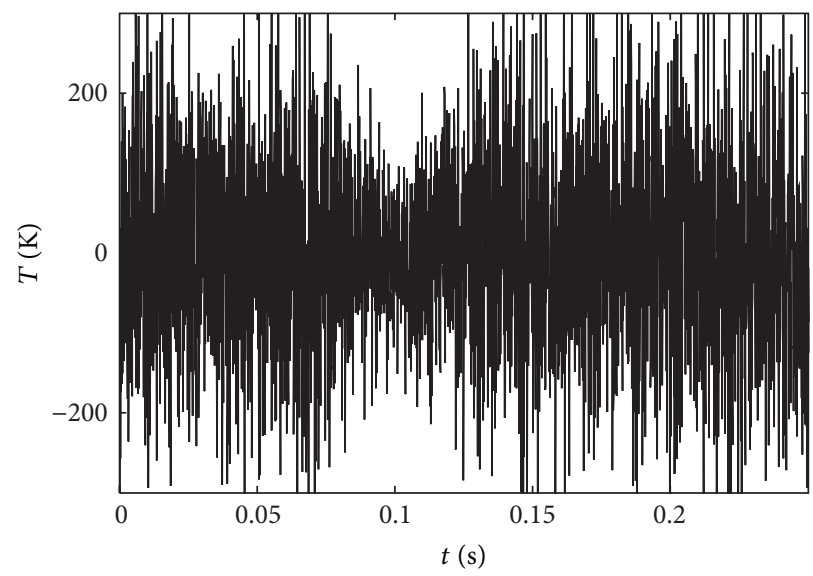

(c) Input signal of pond in horizontal polarization

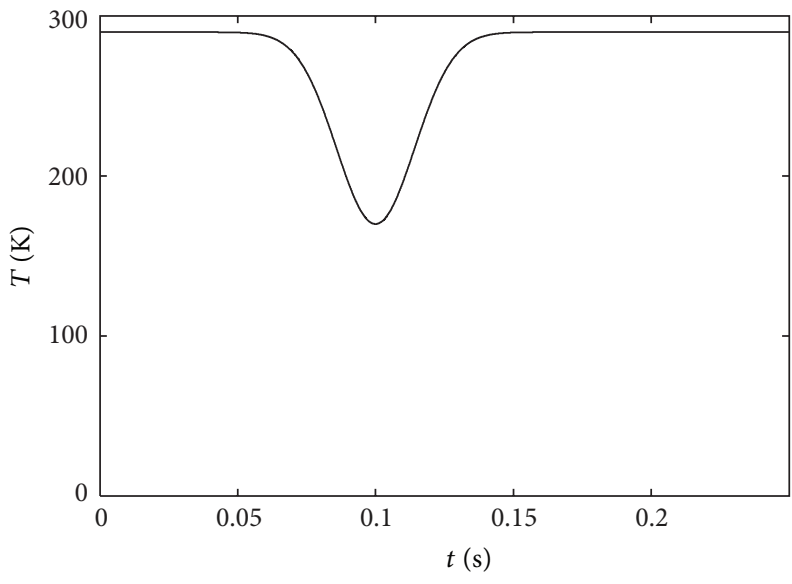

(b) Simulation of horizontal polarization input temperature of pond

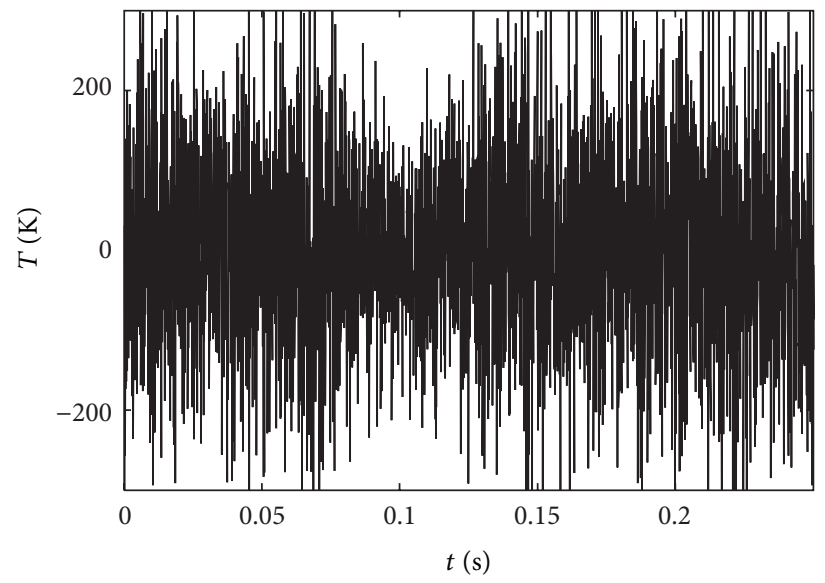

(d) Input signal of pond in horizontal polarization

FIGURE 6: RF input signal of pond.

\section{The Solution of Inconformity in Gain}

According to above analysis, we can get that system detects and locates target and identifies false target through output signal SNR. If output signal range is higher than threshold, then the target which is detected by system is real target; otherwise, it is false target.

In ideal conditions, the polarization difference in temperature of dual-polarization MMW radiometer with dualchannel designed in the literature [4] is $0 \mathrm{~K}$ to real target metal, $30 \mathrm{k}$ to false target pond. So, the false dismissal probability which is got from the diagram on which homologous detection probability varies with SNR is less than 1\%; the probability of eliminating the false of the system is more than $99 \%$. But in the actual situation, the gain of the receiver can not be in full accord through existing technology, so that the system performance of the dual-polarization radiometer which adopts two receivers will be serious by effected also, erroneous judgment will occur. Below is concrete analysis.

According to physical truth, the inconsistency of the gain of receiver is $10 \%$. Due to the effect of gain inconformity, when detecting pond, the output SNR from subtracter is
SNR $=6$ according to the literature [6]. That means difference in temperature of polarization is $\Delta T=15 \mathrm{~K}$; at this time, probability of distinguishing false target is reduced to $90 \%$.

In a similar way, when metal target is detected in fact, the output SNR from subtracter is $\mathrm{SNR}=8$; that means difference in temperature of polarization is $\Delta T=20 \mathrm{~K}$; false dismissal probability of real target is more than $50 \%$.

From above analysis, that in actual situation, it is shown that due to inconsistency of the gain of receiver, recognition performance of dual-polarization radiometer with dual channel will become worse. So, the study of reducing two channels to one channel is very important. The radiometer system designed in this paper does not have the above problems because only one receiver is used. Probability of eliminating the false will be more than $99 \%$ and false dismissal probability is less than $1 \%$ through choosing threshold reasonably.

\section{Simulation Results}

To prove the feasibility of the theory, dual-polarization MMW radiometer with dual-channels and the MMW system 


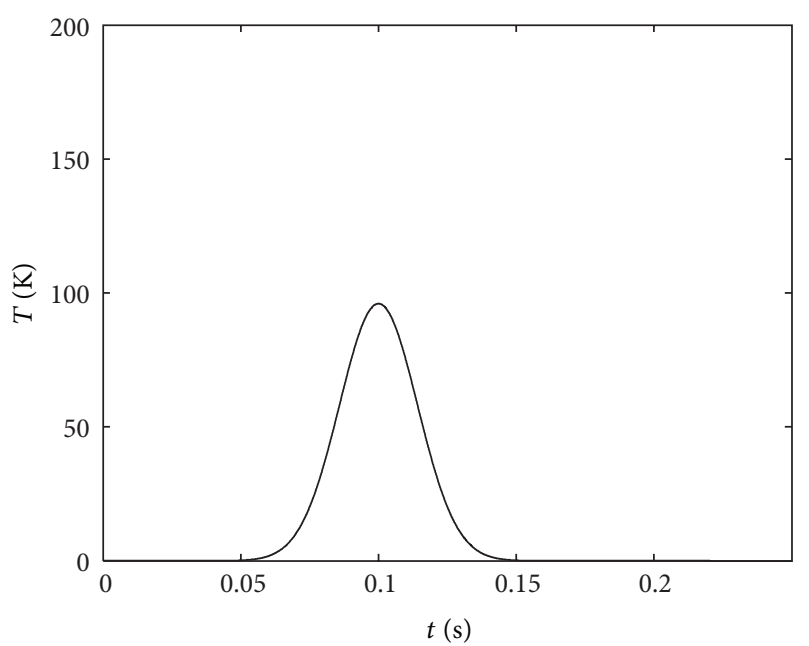

(a) Result of horizontal polarization channel

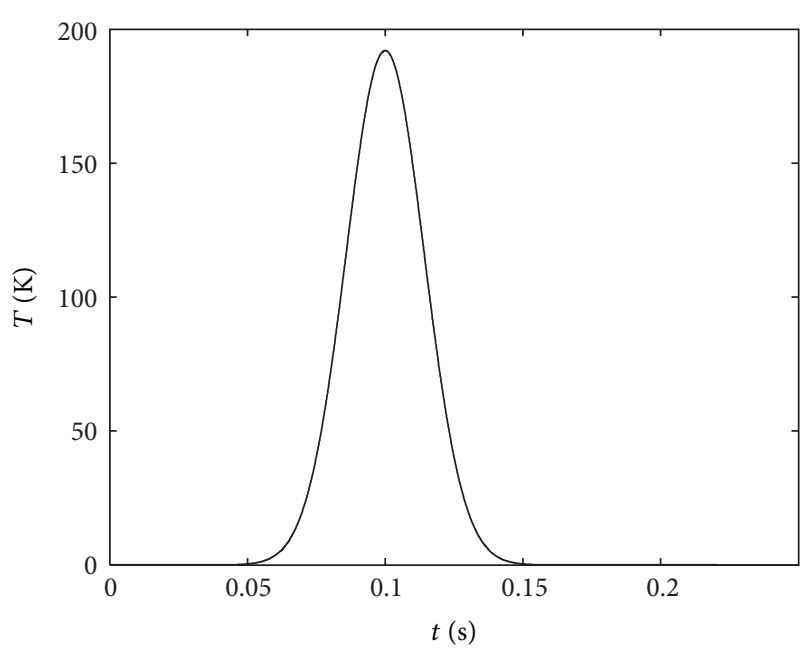

(c) Addtion result of two-way signals

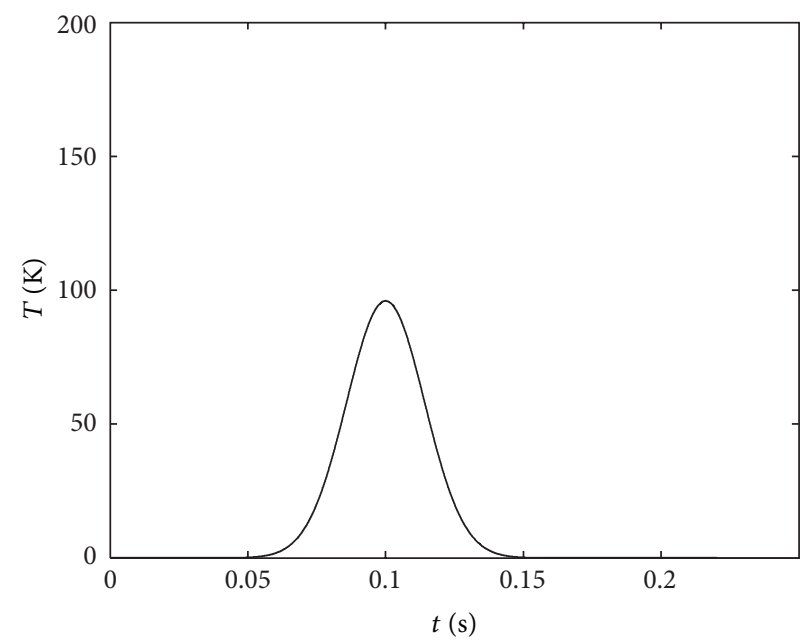

(b) Result of vertical polarization channel

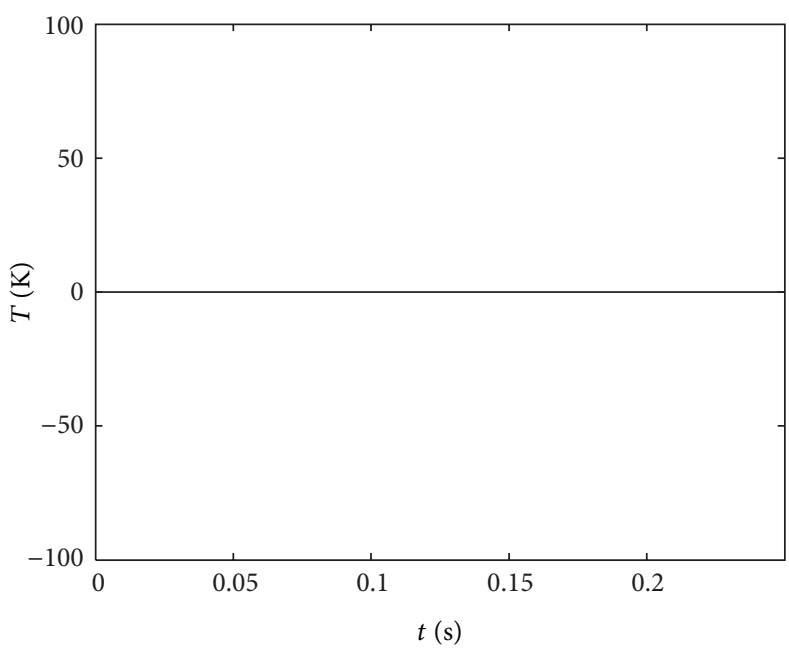

(d) Subtraction result of two-way signals

Figure 7: Output result when detecting metal.

designed in this paper are simulated; then, the simulation results are compared. First of all, band-limited Gaussian white noise is produced, as shown in Figure 4 . Because the operand of $34 \mathrm{GHZ}$ noisy signal can not be supported by simulated program, noisy signal frequency used is reduced to $10^{5} \mathrm{~Hz}$, with $10^{4} \mathrm{~Hz}$ bandwidth.

Then, it will produce a MMW signal collecting cycle with $4 \mathrm{rps}$; finally, it produces RF input signal of metal target and pond by simulation together with noisy signal, as shown in Figures 5 and 6.

5.1. Simulation Result of Dual-Polarization Radiometer with Dual Channels. Metal target output result will be gotten after amplification, detection, integration, and opposition through receiver, as shown in Figures 7(a) and 7(b). At last, two-way signals will be added and subtracted in signal processor; the result is shown in Figures 7(c) and 7(d). We can get that if metal target's horizontal polarization signal and vertical polarization signal were added together, then a great crest signal will be outputted; if subtracted from each other, output is zero.

In a similar way, we can get output result of signal processor when detecting pond, as shown in Figure 8.

According to Figure 8, a crest is outputted after subtracting between pond's horizontal polarization and vertical polarization signal, so we can identify false target through judging output SNR subtracter.

\subsection{Simulation Result of Radiometer System That This Paper} Designed. After metal target and pond RF input signal phasing shift $180^{\circ}$ and power dividing, we can get the signal of metal target inputting receiver and the signal of pond inputting receiver, as shown in Figure 9.

The output result will be got after amplification, detection, integration, and opposition through receiver; the final output result is shown in Figure 10.

We can get form Figure 10 that metal target outputs a higher crest signal and false target pond outputs a lower 


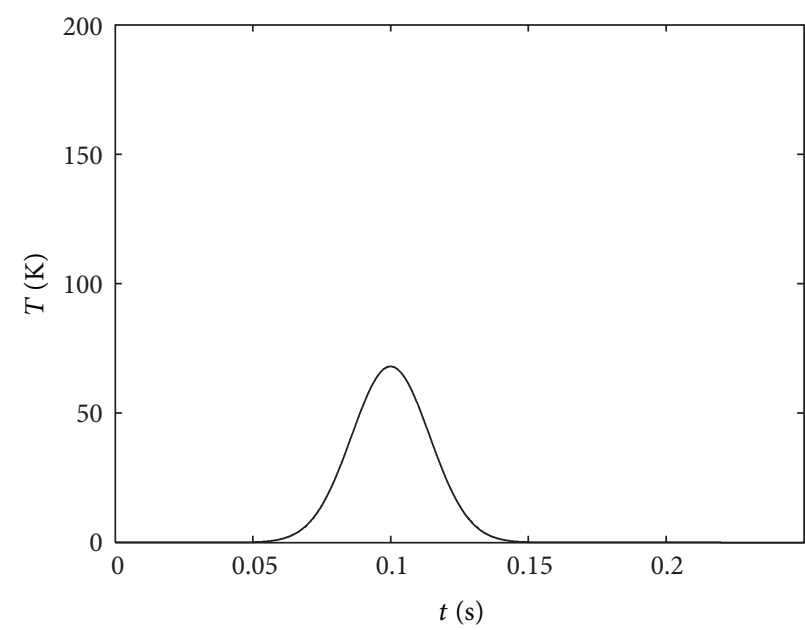

(a) Result of horizontal polarization channel

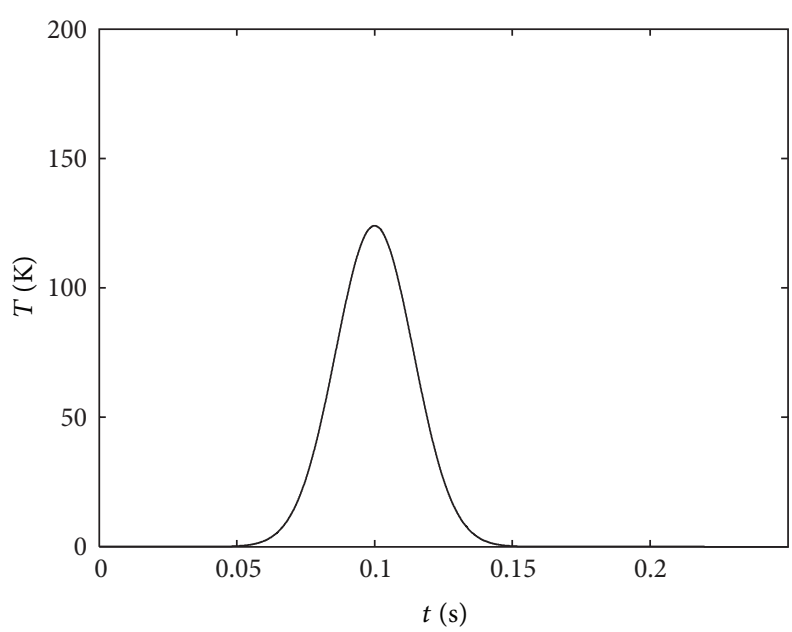

(c) Addition result of two-way signals

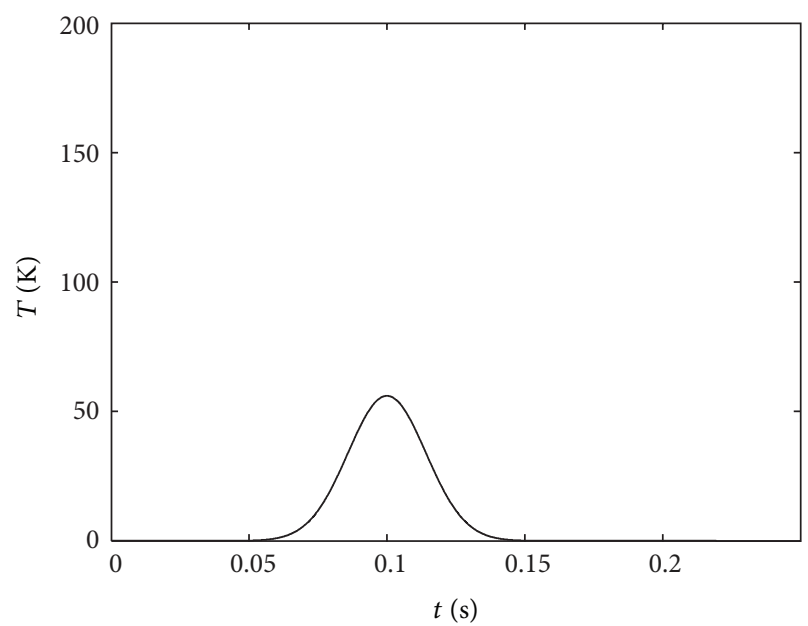

(b) Result of vertical polarization channel

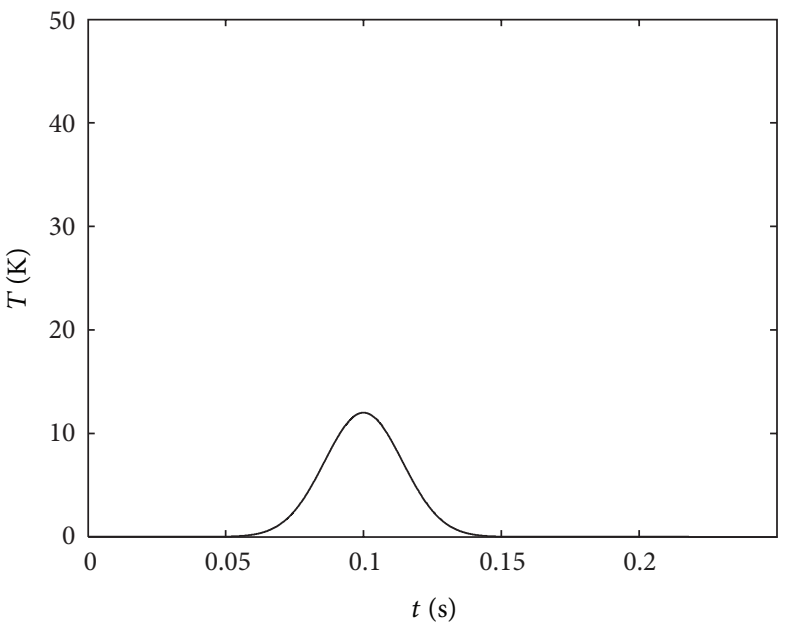

(d) Subtraction result of two-way signals

Figure 8: Output result when detecting pond.

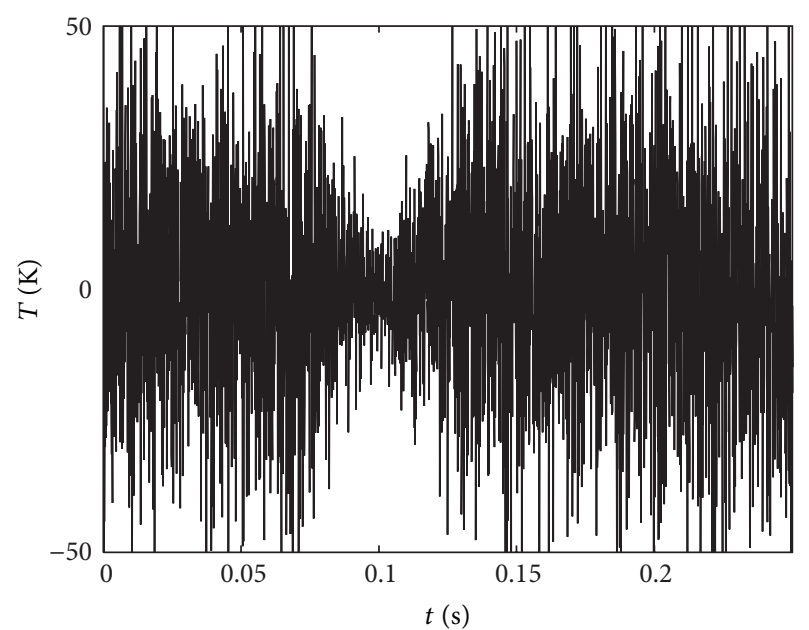

(a) Input signal of receiver by metal target

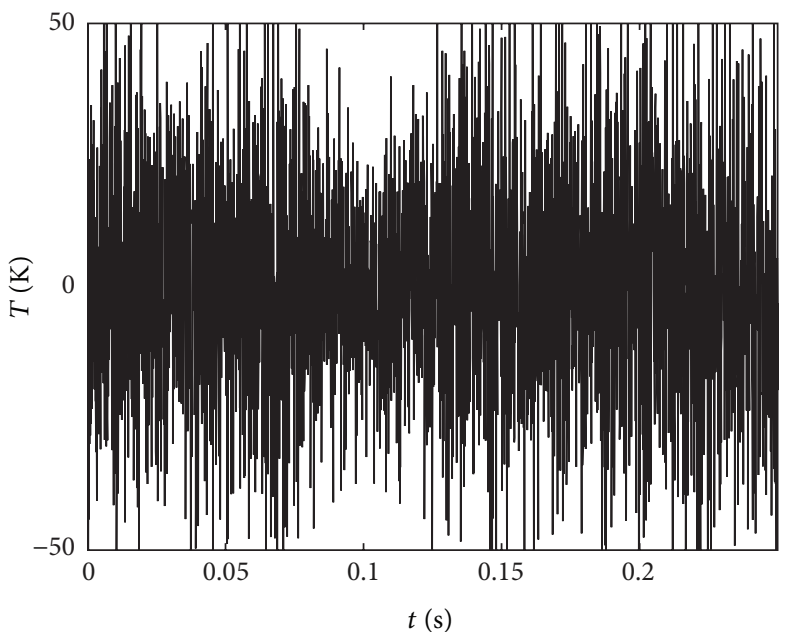

(b) Input signal of receiver by pond

FIGURE 9: Input signal of receiver. 


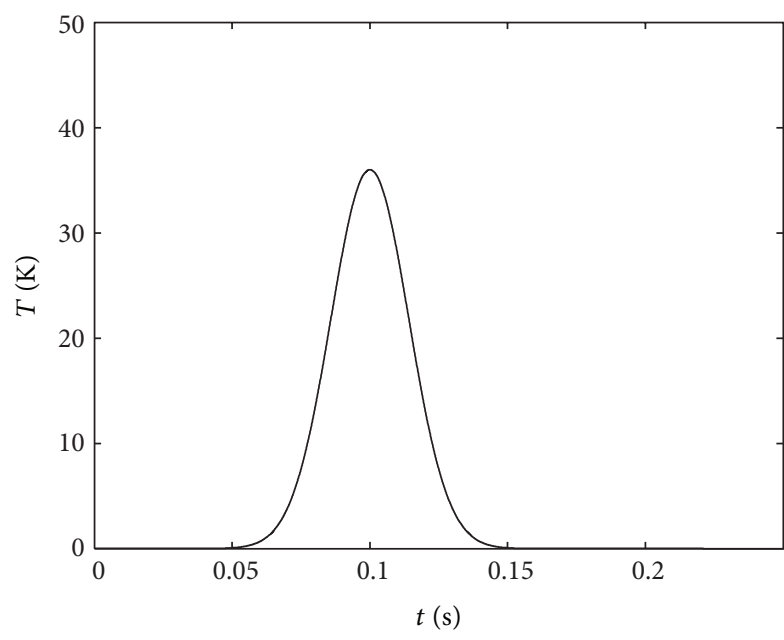

(a) Output temperature of metal target

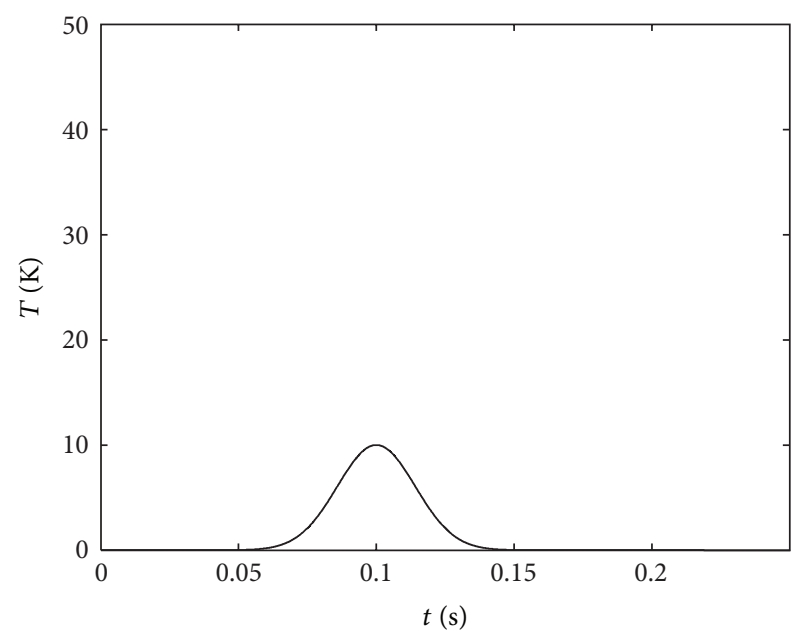

(b) Output temperature of pond

Figure 10: Result of single channel.

crest signal. Also we can posit and judge the output signal according to setting threshold and identify whether the target is false or not.

\section{Conclusion}

This paper designs a dual-polarization total power MMW radiometer with single channel. It can solve the problem of recognition performance of dual-polarization radiometer with dual channels becoming worse caused by the inconformity of gain of receiver. It has a recognition performance on the false target which has great difference on the apparent temperature of horizontal and vertical polarization. Meanwhile, this system just adopt one-way direct-detection receiver; it is small in size and has low cost. It saves summator and related hardware when dealing with back-end signal processing. Validity of this theory at last is proved by simulation result.

\section{Conflict of Interests}

The authors declare that there is no conflict of interests regarding the publication of this paper.

\section{References}

[1] Z.-Y. Zhang, W. Guo, and S.-J. Lin, "A passive mm-wave sensorfused short," Journal of Huazhong University of Science and Technology, vol. 22, no. 5, pp. 20-25, 1994.

[2] J.-J. Xie, T.-F. Wu, and W.-C. Wang, "Applications of detection technology of millimeter wave in intelligent mine," Guidance and Fuze, vol. 27, no. 2, pp. 56-60, 2006.

[3] Q. Li, "Microwave brightness temperature prediction of plane targets by a neural network," IEEE Transactions on Geoscience and Remote Sensing, vol. 41, no. 1, pp. 160-162, 2003.

[4] Y.-H. Zhang and W. Wu, "Research on Ka-band dualpolarization direct detection total power radiometer," Acta Armamentarii, vol. 30, no. 3, pp. 375-379, 2009.
[5] X. Mei, A Dual-Polarized Microstrip Antenna, Nanjing University of Science and Technology, 2008.

[6] Y.-H. Zhang, The research of key techniques in passive millimeterwave dual-polarization detecting and phased array beam steering [Ph.D. thesis], Nanjing University of Science and Technology, 2010.

[7] W. E. I. Qian-hua, A thesis submitted in partial fulfillment of the requirements for the degree of master of engineering [M.S. thesis], Huazhong University of Science and Technology, 2004.

[8] Z. Sheng, S.-Q. Xie, and C.-Y. Pan, Probability and Mathematical Statistics, Higher Education Press, Beijing, China, 2001.

[9] L.-F. Ding, Principle of Radar, Publishing House of Electronics Industry, Beijing, China, 2009. 

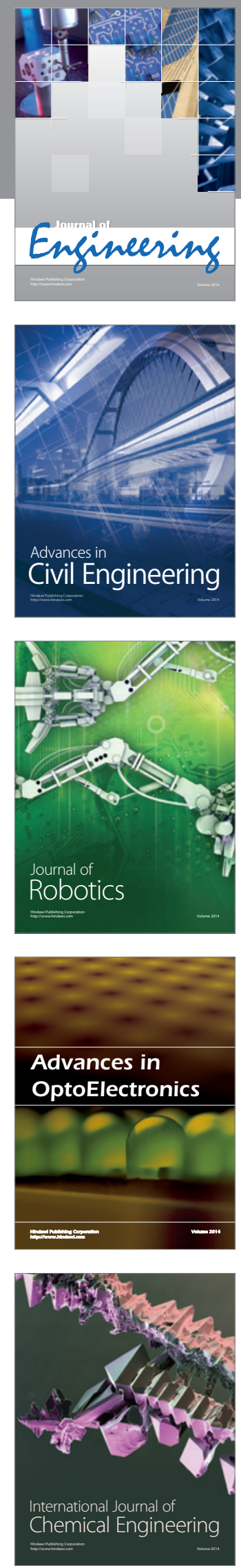

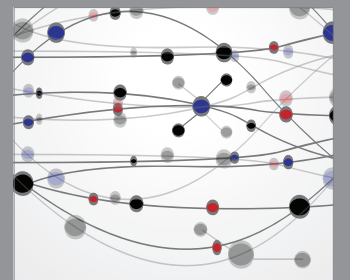

The Scientific World Journal
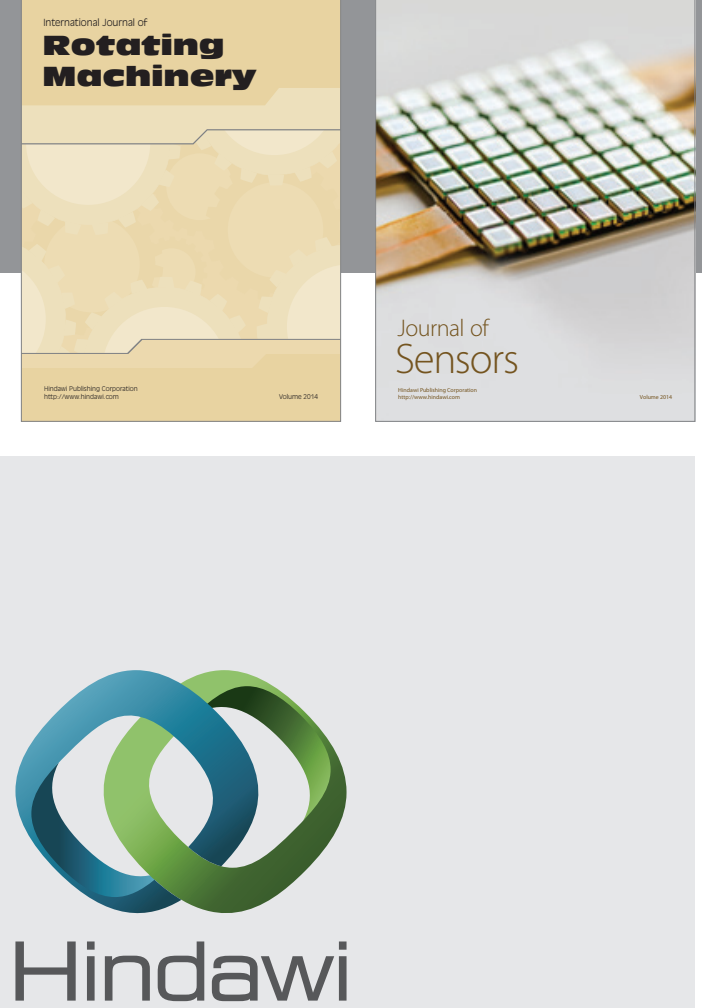

Submit your manuscripts at http://www.hindawi.com
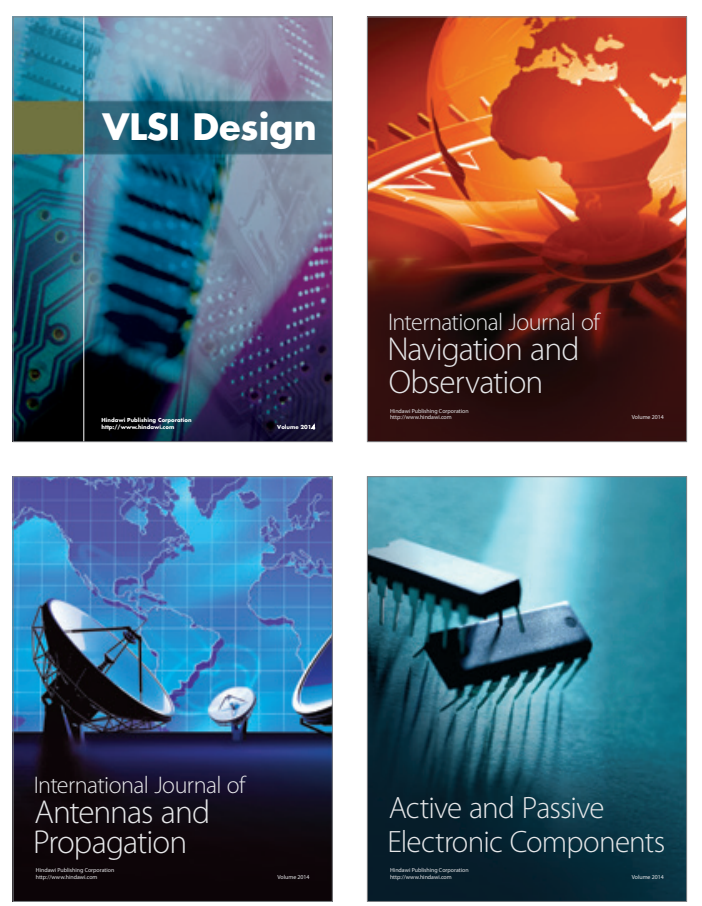
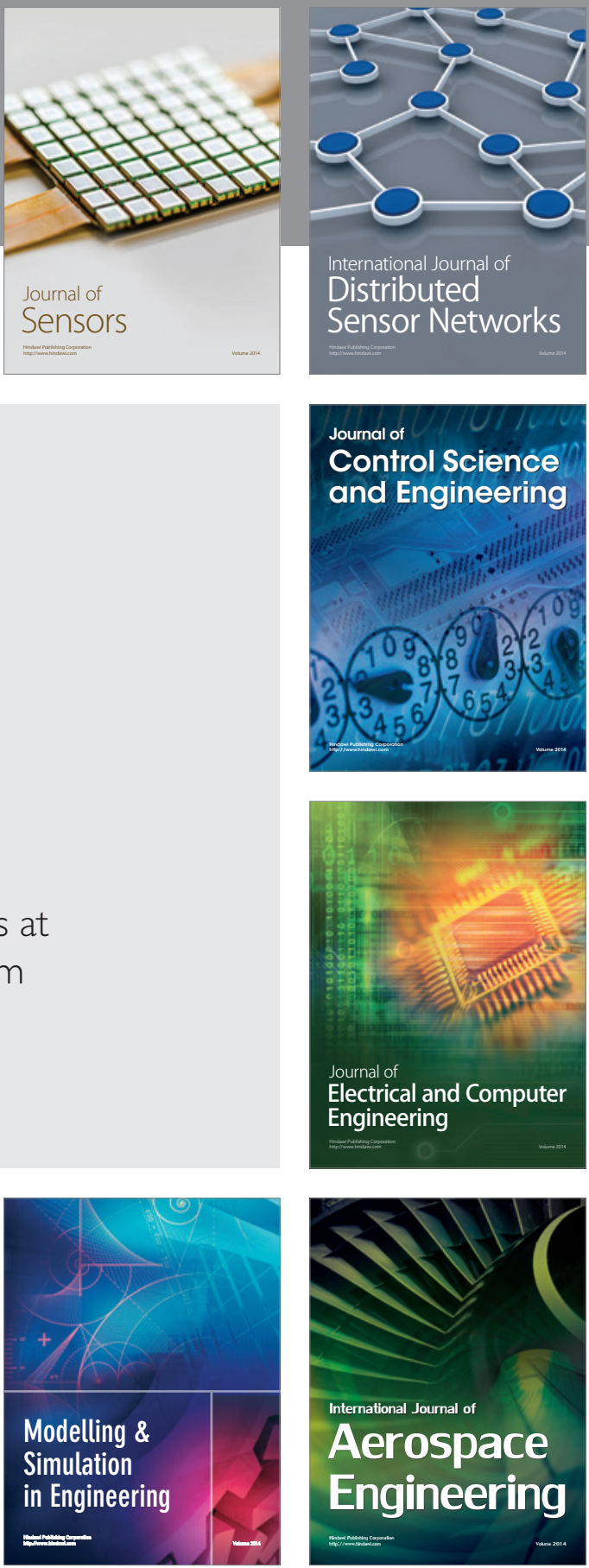

Journal of

Control Science

and Engineering
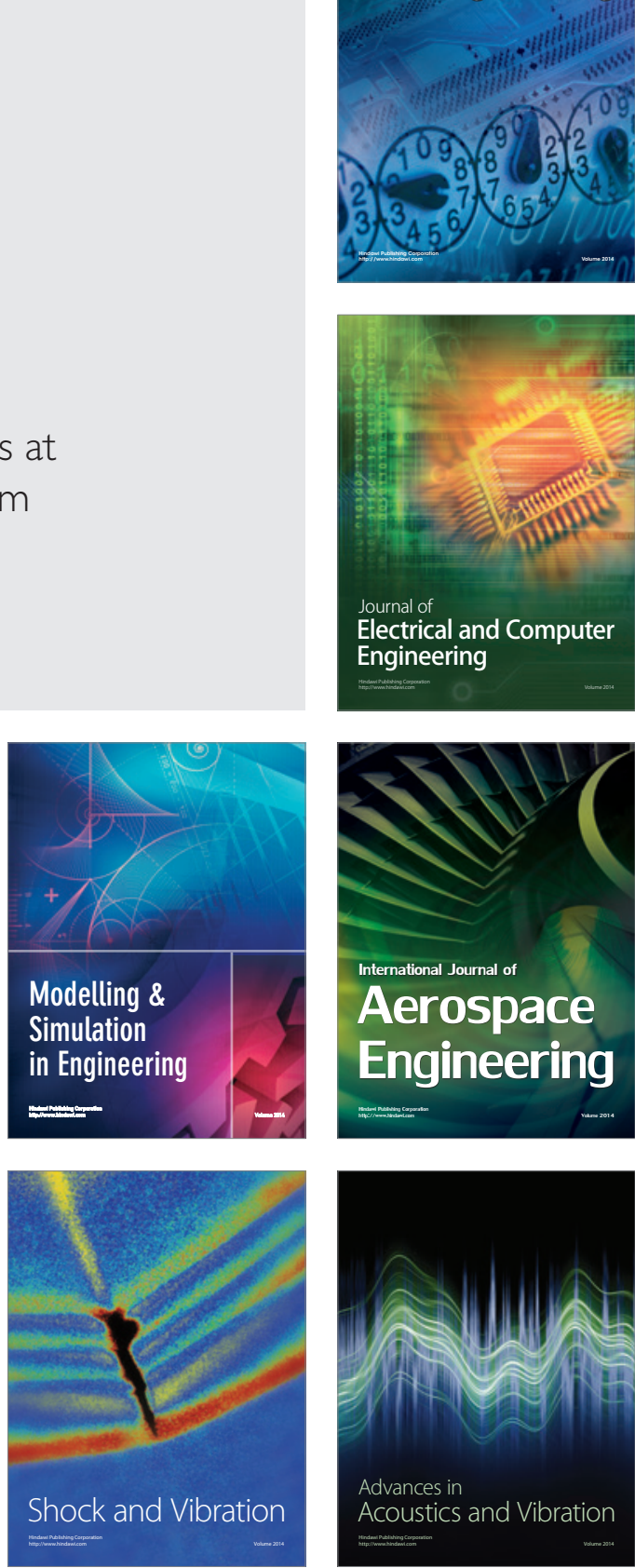